\title{
FACTORES INTERNOS Y EXTERNOS RELACIONADOS CON LA ROE Y ROA DE BANCOS PRIVADOS ECUATORIANOS DESDE LA DOLARIZACIÓN
}

\section{INTERNAL AND EXTERNAL FACTORS RELATED TO THE ROE AND ROA OF ECUADORIAN PRIVATE BANKS SINCE DOLLARIZATION}

\author{
Gylson Jara Quevedo ${ }^{1}$, Oswaldo Massuh Arreaga ${ }^{2}$, Alex Ibarra Velásquez ${ }^{3}$, José \\ Castro Carrasco ${ }^{4}$, Stalin Zurita Vargas ${ }^{5}$, Adrian Mendoza Malo ${ }^{6}$
}

\author{
Palabras clave: \\ Rentabilidad \\ banca privada \\ factores externos \\ factores internos
}

\begin{abstract}
Resumen
Posterior a la crisis financiera de finales de los años 90, el Ecuador evidenció una notable estabilidad económica desde la adopción del dólar como moneda de curso legal y las políticas monetarias implementadas, además, la banca privada ecuatoriana presentó un constante crecimiento influenciado por diversos factores. Ante este contexto, la presente investigación analizó la relación que han tenido como factores internos las cuentas de bancos privados, sus indicadores de gestión, medidas de concentración en el sector bancario y como factores externos variables macroeconómicas en relación con la rentabilidad en términos de ROE y ROA de los bancos privados ecuatorianos de acuerdo con su tamaño desde la dolarización instaurada en el país. Mediante regresiones econométricas con información extraída de fuentes oficiales establecida bajo la estructura de datos de panel se identificaron como factores internos relacionados con las medidas de rentabilidad a las cuentas por pagar, las cuentas por cobrar las inversiones, índices como la capacidad comercial, la liquidez, el apalancamiento, además de la concentración de créditos y de depósitos. Entre los factores externos el PIB y el precio del barril, de petróleo resultaron ser las variables macroeconómicas más relevantes en torno de las medidas de rentabilidad de los bancos privados.
\end{abstract}

Códigos JEL: C33, G01

Keywords: Profitability, private bank, internal factors, external factors

\footnotetext{
${ }^{1}$ Ministerio de Agricultura y Ganadería, Quito, (Ecuador).

E-mail: gylxavi@gmail.com

${ }^{2}$ Ministerio de Agricultura y Ganadería, Quito, (Ecuador).

E-mail: oswaldomassuh@gmail.com

${ }^{3}$ Universidad Agraria del Ecuador, Guayaquil, (Ecuador).

E-mail: aibarra@uagraria.edu.ec

${ }^{4}$ Escuela Politécnica del Litoral, Guayaquil, (Ecuador).

E-mail: jcastro@espol.edu.ec

${ }^{5}$ Universidad Agraria del Ecuador, Guayaquil, (Ecuador).

E-mail: szurita@uagraria.edu.ec

${ }^{6}$ Naturisa S.A, Guayaquil, (Ecuador).

E-mail: octavio43.98@gmail.com
} 
Keywords:
Profitability
private bank
internal factors
external factors

\section{INTRODUCCIÓN}

En la actualidad, el sistema financiero ecuatoriano es más estable y brinda confianza entre los usuarios. Según la Asamblea Nacional Constituyente del Ecuador (2008), este sistema se conforma por entidades públicas, privadas y de economía popular y solidaria, además de instituciones de control cuyo fin es el óptimo crecimiento económico del país.

No obstante, antes del inicio de presente siglo La Ley General de Instituciones del Sistema Financiero (Registro Oficial No. 475, 1994). La instauración de esta ley flexibilizó las normas que regulaban la creación y el manejo de entidades bancarias, las cuales otorgaban créditos sin controles en cuanto a las tasas de interés y las operaciones off-shore que realizaban. Esto ocasionó la insolvencia y falta de liquidez de instituciones bancarias, dando lugar a la crisis económica del Ecuador en 1999, cuyas consecuencias se vieron reflejadas en exorbitantes niveles de inflación, el congelamiento y disolución de los fondos existentes en las instituciones financieras y el cierre de bancos en el país (Romero A., 2004-2005).
Ante esta situación, el 13 de marzo del 2000, el Congreso Nacional (2000) decretó la Ley Para La Transformación Económica del Ecuador que oficialmente deja al Sucre fuera de circulación y adopta el dólar norteamericano como única moneda en curso en el territorio ecuatoriano.

Como resultado, el dólar trajo ventajas a la economía nacional, debido a que se evidenció una disminución de los niveles de inflación gracias a la estabilización de precios, el incremento progresivo de las cifras del PIB nacional y mayor estabilidad en cuanto a ingresos petroleros e inversión pública (Toscanini, et al., 2020).

Desde entonces, la banca nacional se ha mantenido en constante evolución y estabilidad, puesto que las ganancias generadas por las instituciones bancarias han permitido su permanencia como parte del sistema financiero, contribuyendo al crecimiento económico del país (Pampillón, et al., 2012).

Cabe destacar que un aspecto importante de la industria bancaria es su estabilidad, por lo que son diversos los factores internos y externos que influyen en su desempeño. Por una parte, los factores internos están vinculados con la gestión 
interna de cada banco y las decisiones en cuanto a sus indicadores y niveles de riesgo; mientras que los factores externos están más relacionados con la concentración del mercado y el ámbito macroeconómico del país (Curak, et al., 2012).

En este contexto, la presente investigación tiene como objetivo identificar la relación entre factores de las instituciones bancarias como sus cuentas de indicadores de operaciones, niveles de concentración de mercado y variables macroeconómicas respecto de los indicadores de rentabilidad sobre el patrimonio (ROE) y sobre activos (ROA) de los bancos privados del Ecuador según su tamaño, desde que se estableció la dolarización en el Ecuador (2000 - 2020).

\section{El sistema financiero ecuatoriano y la rentabilidad de la banca}

A nivel mundial el sistema financiero se conforma por entidades públicas y privadas reguladas por un banco central $\mathrm{u}$ organismos de control bancario y financiero (Noboa S., 2008). Chiriboga (2010) sostiene que la importancia del sistema financiero radica en que por medio de éste se facilita el acceso a servicios de pago, movilización de fondos en forma de ahorro y la asignación de créditos, también permite compartir riesgos entre instituciones que incursionan en diversas actividades.

En el Ecuador, el ente regulador de las instituciones bancarias es la Superintendencia de Bancos, esta reconoce como entidades privadas del sistema financiero a bancos, cooperativas de ahorro y crédito, sociedades financieras, $\mathrm{y}$ mutualistas. Además, como se detalla en la TABLA 1, clasifica por tamaño a los bancos de acuerdo con la participación de sus activos en el total del sector, categorizando como bancos pequeños a las instituciones con una participación menor o igual al 12\%, medianos con menos del $36 \%$ y grandes con una participación mayor al 36\% (Cruz Rodríguez, 2015).

Los bancos grandes son pocos en contraste con los medianos y pequeños; no obstante, tienen mayor participación de mercado (Uzcátegui-Sánchez, et al., 2018). Además, como lo indican Jara, Sánchez, Bucaram y García (2018) los bancos pequeños en el período 2000-2017 presentan en su mayoría niveles medios de concentración de mercado, considerando que el número de instituciones privadas nacionales oscila entre 8 y 13 bancos.

TABLA 1

Bancos privados del Ecuador, a diciembre del 2020

\begin{tabular}{lll}
\hline PEQUEÑOS & MEDIANOS & GRANDES \\
\hline Amazonas & & \\
Bancodesarrollo & Austro & \\
Capital & Bolivariano & \\
Comercial de & Citibank & \\
Manabí & Diners & Guayaquil \\
Coopnacional & General & Pacífico \\
Delbank & Rumiñahui & Pichincha \\
D-Miro S.A. & Internacional & Produbanco \\
Finca & Loja & \\
Litoral & Machala & \\
Procredit & Solidario & \\
Visionfund Ecuador & & \\
\hline
\end{tabular}

Así también, Baumol y Blinder (2009) establecen que los bancos reciben depósitos por parte del público sin ninguna tasa de interés, consecutivamente los invierten mediante préstamos con una tasa fijada y así obtienen ganancias como cualquier otro negocio. Dichas inversiones benefician a diferentes sectores productivos del país, como es el caso del sector agropecuario ecuatoriano, que a través de recursos económicos inyectados por las instituciones financieras puede lograr el incremento de su productividad y expansión de mercado (Valdez M., et al., 2020).

En el Ecuador, las principales medidas de rentabilidad son el Rendimiento sobre el Patrimonio (ROE) y sobre Activos (ROA). Al desarrollar sus operaciones en una economía dolarizada los índices de rentabilidad de los bancos ecuatorianos pueden incrementarse, debido a que el dólar, en comparación con otras divisas, permite reducir los niveles de incertidumbre respecto del riesgo cambiario indirecto (Vera-Gilces, et al., 2020). 


\section{Estado del arte}

Existen algunos estudios que evalúan los factores que inciden en la rentabilidad de la banca, entre estos se destaca el trabajo de Athanasoglou, Brissimis, y Delis (2008), quienes analizaron la ROE y ROA de la industria bancaria en Grecia, concluyendo que variables macroeconómicas como la inflación, afectan el rendimiento del sector bancario.

García-Herrero, Gavilá, y Santabárbara (2009), en China, analizaron con una muestra de 87 bancos, entre los años 1997 a 2004, los bajos niveles de ROA de estos. A partir de ello, evidenciaron que los préstamos vencidos fueron los principales determinantes de la rentabilidad. Además, identificaron que el bajo crecimiento económico del país tenía fuerte influencia en el rendimiento de los bancos y que las variables internas de los bancos no se veían afectadas por la inflación.

En el estudio realizado en Macedonia por Curak, Poposki y Pepur (2012) se analizaron determinantes de la rentabilidad bancaria respecto de factores específicos de los bancos, de la industria bancaria y factores macroeconómicos. Concluyeron que la rentabilidad de los bancos está determinada principalmente por factores internos como la gestión de los gastos operativos, los niveles de riesgo de solvencia y de liquidez. A su vez, identificaron que los niveles de concentración de mercado son relevantes para la rentabilidad de los bancos y que el PIB era un factor determinante de rentabilidad.

Por otro lado, Abduh y Omar (2013), en Malasia, analizaron bancos convencionales e islámicos durante el período 2005-2009 a fin de identificar los determinantes de rentabilidad empleando factores internos de los bancos y variables macroeconómicas. En el estudio se concluyó que factores como la liquidez y el PIB están relacionado con la evolución de la ROA de los bancos, demostrando que la evolución del PIB del país tuvo una fuerte influencia con la rentabilidad bancaria.

Así mismo, Osuagwu (2014) en Nigeria tomando como muestra información anual de los bancos como variables propias de los bancos y de la industria bancaria, además información macroeconómica desde 1980 a 2010. De esto se demostró que las variables propias de los bancos como los depósitos y préstamos están altamente relacionadas con la rentabilidad. Así mismo, la inflación y el tipo de cambio fueron variables determinantes en la rentabilidad de los bancos.

Mediante estimaciones con datos de panel, Chidozie y Ayadi (2017) evidenciaron que el crecimiento del PIB no afecta la rentabilidad bancaria en Nigeria. Además, su estudio concluyó que el precio del barril de petróleo afecta la rentabilidad del sector financiero.

Ashraf, Haider, y Sarwar (2017), mediante un análisis de datos de panel con muestras de bancos en Asia, evaluaron los efectos que tienen en la rentabilidad bancaria, el PIB, las tasas de inflación, desempleo, el tipo de cambio, el precio del barril de petróleo y la concentración de mercado; demostrando que la liquidez (factor interno) y el crecimiento del PIB (factor externo) poseían alta significancia en relación con la rentabilidad de los bancos.

En Ecuador, Jara Quevedo et al., (2018) analizaron la rentabilidad de pequeños bancos privados desde la dolarización; concluyendo que factores propios de los bancos como depósitos a plazo, los créditos y las ratios de apalancamiento e intermediación financiera influyen en la rentabilidad de los pequeños bancos. Desde la perspectiva macroeconómica, estos afectan la rentabilidad del PIB y el índice de inflación.

Así mismo, en el estudio de Camino-Mogro \& Armijos-Bravo (2018) se analizó las condiciones de competencia del mercado de la banca privada ecuatoriana entre el año 2000 a 2015, concluyendo que en dicho período los bancos privados tenían una competencia de mercado monopolística. No obstante, la competencia cambió entre 2007 y 2015, puesto que las regulaciones evitaron colusiones entre competidores.

Por otro lado, Romero, Flores, Reyes y Campoverde (2020) analizaron la concentración y competencia en el mercado bancario ecuatoriano 
aplicando el índice de Herfindahl-Hirschman, el índice de Lerner, el Índice de Concentración y el Índice de Dominancia a las cuentas de los bancos como el capital de los bancos, la cartera de crédito, depósitos y las inversiones durante los años entre 2006 y 2018. Es así como concluyeron que el sector bancario privado no presenta niveles de concentración o una competencia monopolística.

Por último, Solano, Camino-Mogro y Armijos-Bravo (2020) analizaron la competitividad del sector bancario ecuatoriano, clasificando las instituciones según el tamaño, mediante una estructura de datos de panel usando el modelo de mínimos cuadrados generalizados factibles y panel de corrección de errores estándar como modelos alternativos. En el estudio no se encontró evidencia de un mercado no competitivo entre los bancos medianos y los pequeños, siendo que compiten en el sector como en competencia monopolística.

\section{METODOLOGÍA}

Para el desarrollo del presente estudio se extrajo información financiera oficial de la Superintendencia de Bancos y el Banco Central del Ecuador, respecto de los balances de las instituciones que conforman el sector de la banca privada, sus indicadores de rendimiento $\mathrm{y}$ variables macroeconómicas durante el período comprendido desde el 2000 al 2020. Esta información fue contrastada con otras fuentes como de la Asociación de Bancos del Ecuador (ASOBANCA).

Con dicha información se generó un conjunto de datos de panel y se ejecutó un análisis orientado a cuantificar la relación existente entre las medidas de rentabilidad de los bancos privados y cómo éstas son influenciadas por el desempeño de determinadas cuentas e indicadores inherentes a la banca privada y variables macroeconómicas, durante el período de estudio. Además, se analizaron los niveles de concentración de mercado mediante el Índice de Herfindahl Hirschman. Para ello, se utilizó el software econométrico gretl.

\section{Variables dependientes}

En este estudio se establecieron como variables dependientes a la Rentabilidad sobre activos (ROA) y la rentabilidad sobre el patrimonio (ROE). La primera está relacionada con las utilidades sobre el total de activos de las organizaciones y la segunda es la rentabilidad financiera, la cual se obtiene de la relación de las utilidades sobre el patrimonio de las entidades (García O., et al., 2018).

\section{Variables independientes}

Como variables independientes, se consideraron las cuentas declaradas según la Superintendencia de Bancos (2017):

- Inversiones (INVERS): abarcan montos invertidos por los bancos a fin de generar reservas de liquidez.

- Cartera de Créditos (CAR_CRED): engloba los créditos que otorgan los bancos.

- Cuentas por Cobrar (CTAS_COBR): representan valores de cobro inmediato para los bancos por diferentes conceptos.

- Depósitos a la vista (DEP_VISTA) y a plazo (DEP_PLAZO): son cuentas de pasivo, los primeros son depósitos que reciben los bancos por menos de 30 días, los otros son exigibles a más de 30 días (Superintendencia de Bancos, 2021).

- Cuentas por Pagar (CTASPAGR): registran valores pendientes de pago de los bancos.

En cuanto a los indicadores de las actividades de los bancos, se consideraron:

- Intermediación Financiera (INTERM_FIN): proporción en que los depósitos de los bancos son colocados mediante créditos en diferentes ámbitos económicos, para generar ingresos por los intereses (ASOBANCA, 2017).

- Apalancamiento (APALANC): nivel de endeudamiento de las entidades bancarias que es financiado con fondos del patrimonio (Gibson, 2012).

- Capacidad Comercial (CAPAC_COMERC): mide la capacidad 
que tienen los bancos para obtener ganancias por el otorgamiento de créditos, este indicador relaciona los valores de la cartera total sobre los activos totales (Gómez G., et al., 2009).

- Índice de Liquidez (LIQUIDEZ): muestra la capacidad de las entidades para obtener ingresos mediante la inversión en activos, esta medida se obtiene de la relación de los fondos disponibles y el total de depósitos a plazo (ASOBANCA, 2017).

- Concentración de Depósitos Totales (HHI_DEPOS): considera el nivel de concentración de mercado de los depósitos totales de los bancos del sector.

- Cartera de Créditos (HHI_CAR_CRED) de las instituciones financieras.

En torno a las variables macroeconómicas, se acogieron las siguientes:

- Inflación (INFLAC): nivel de variación de precios en la economía de un período a otro (Jones, 2009 ).

- Riesgo país (RIESGO_P): es la capacidad que tiene el país para resolver conflictos financieros a nivel macroeconómico y político (Alvarado V., 2016).

- Producto Interno Bruto (PIB_REAL): muestra la sumatoria del valor de mercado de los productos finales y los servicios generados durante un período específico, determinando la relación que existe entre el nivel de vida de los habitantes con la productividad nacional (Parkin, et al., 2007); y su tasa de variación periódica se considerada el principal indicador de la evolución de la economía del país (BCE, 2021).

- Valor del Precio anual del barril de petróleo ecuatoriano de exportación (P_PETROL) a diciembre de cada año.

En el apéndice A se resumen las variables a utilizar en el análisis econométrico.

\section{Modelo econométrico}

El análisis econométrico se realizó mediante Mínimos Cuadrados Ordinarios (MCO) empleando la estructura de datos de panel, aplicando logaritmos a las variables. El modelo de análisis para las estimaciones será el propuesto por Athanasoglou (2008), analizado por Dietrich y Wanzenried (2010), y citado por Osuagwu (2014) que está definido de la siguiente manera:

$$
\Pi_{i t}=c+\Pi_{i, t-1}+\sum_{j=1}^{J} \beta_{j} X_{i t}^{j}+\sum_{l=1}^{L} \beta_{l} X_{i t}^{l}+\varepsilon_{i t}
$$

Donde $\Pi_{i t}$ corresponde a la rentabilidad de los bancos $\mathrm{i}$ en el tiempo t; $c$ es la constante paramétrica; $\Pi_{i, t-1}$ es la rentabilidad de los bancos $i$ con un rezago $\mathrm{t}-1 ; X_{i t}$ con los superíndices j y 1 denotan los factores específicos de los bancos y las variables macroeconómicas respectivamente; $\varepsilon_{i t}$ Indica el término de error estocástico.

\section{RESULTADOS}

Los resultados del análisis econométrico se obtuvieron mediante el software econométrico Gretl, considerando datos indizados por los años del período de estudio y cada banco como observación considerando su tamaño como categoría. En la ¡Error! No se encuentra el origen de la referencia. se muestran los estadísticos principales de las variables en estudio, a las cuales se les ha añadido logaritmos naturales, a fin de estandarizar las series y que las mismas estén en la misma magnitud.

TABLA 2

\section{Estadísticos principales de las variables en} estudio

\begin{tabular}{|c|c|c|c|c|c|}
\hline VARIABLE & MEDIA & MEDIANA & MÍN & MÁX & D. T. \\
\hline \multicolumn{6}{|c|}{ Variables dependientes } \\
\hline 1_ROE & $-2,60$ & $-2,20$ & $-10,20$ & $-0,07$ & 1,34 \\
\hline 1_ROA & $-4,76$ & $-4,46$ & $-23,00$ & $-2,29$ & 1,42 \\
\hline \multicolumn{6}{|c|}{ Variables independientes } \\
\hline 1_INVERS & 9,90 & 9,98 & 0,69 & 14,60 & 2,29 \\
\hline 1_CAR_CRED & 11,60 & 11,70 & $-2,42$ & 15,80 & 2,14 \\
\hline 1_CTAS̄_COBR & 8,01 & 7,96 & 2,16 & 12,40 & 1,89 \\
\hline 1_DEP_VISTA & 11,10 & 11,20 & $-2,24$ & 15,80 & 2,34 \\
\hline 1_DEP_PLAZO & 10,70 & 11,10 & 1,95 & 14,80 & 2,24 \\
\hline 1_CTAS_PAGR & 8,82 & 8,72 & 2,79 & 13,20 & 1,90 \\
\hline 1_INTERM_FIN & $-0,26$ & $-0,34$ & $-6,61$ & 3,20 & 0,81 \\
\hline 1_APALANC & 1,82 & 2,06 & $-1,90$ & 4,10 & 0,79 \\
\hline 1_CAPAC_COMERC & $-0,76$ & $-0,60$ & $-12,30$ & $-0,10$ & 0,82 \\
\hline 1_LIQUID_INMED & $-0,71$ & $-0,72$ & $-6,85$ & 10,60 & 1,29 \\
\hline 1_HHI_DEPOS & 0,12 & 0,69 & $-12,50$ & 6,80 & 3,93 \\
\hline 1_HHI_CAR_CRED & 0,24 & 0,77 & $-28,00$ & 6,90 & 3,93 \\
\hline 1_INFLAC & $-2,78$ & $-3,18$ & $-4,49$ & $-0,09$ & 1,13 \\
\hline 1_RIESGO_P & 6,85 & 6,72 & 6,13 & 8,46 & 0,48 \\
\hline 1_PIB_REĀL & 10,90 & 10,90 & 10,50 & 11,20 & 0,22 \\
\hline 1_P_PETROL & 3,87 & 3,88 & 2,66 & 4,66 & 0,55 \\
\hline
\end{tabular}




\section{Regresiones econométricas}

La Tabla 3 muestra los modelos 1,2 y 3 mediante los cuales se realizaron estimaciones de mínimos cuadrados ordinarios empleando como variable dependiente la $\mathrm{ROE}$ de las instituciones financieras de acuerdo con su tamaño. Es así que para los bancos grandes, las variables cuentas por pagar, apalancamiento, capacidad comercial presentaron un nivel de significancia del $5 \%$ en relación a la variable dependiente. Por su parte, la concentración de mercado de la cartera de créditos presenta un coeficiente de correlación negativo, pero con nivel de significancia del $5 \%$. El PIB presentó una relación negativa y un nivel de significancia del 10\%, así como el precio del barril de petróleo.

TABLA 3

MCO tomando la ROE como variable dependiente

\begin{tabular}{|c|c|c|c|c|c|c|}
\hline \multirow[b]{3}{*}{ Variables independientes } & \multicolumn{6}{|c|}{ Variable dependiente: I_ROE } \\
\hline & \multicolumn{2}{|c|}{$\begin{array}{l}\text { BANCOS GRANDES } \\
\text { Modelo 1: }\end{array}$} & \multicolumn{2}{|c|}{$\begin{array}{c}\text { BANCOS MEDIANOS } \\
\text { Modelo } 2\end{array}$} & \multicolumn{2}{|c|}{$\begin{array}{c}\text { BANCOS PEQUEÑOS } \\
\text { Modelo 3: }\end{array}$} \\
\hline & Coef. & valor $p$ & Coef. & valor $p$ & Coef. & valor $p$ \\
\hline const & 50,13 & $0,05(* *)$ & 65,90 & $0,07(*)$ & 11,26 & 0,75 \\
\hline 1_INVERS & 0,27 & 0,34 & $-0,12$ & 0,47 & $-0,011$ & 0,93 \\
\hline 1_CAR_CRED & $-6,04$ & 0,30 & 0,15 & 0,89 & 0,31 & 0,78 \\
\hline 1_CTAS̄_COBR & 0,08 & 0,67 & $-0,42$ & 0,15 & $-0,198$ & $0,04(* *)$ \\
\hline 1_DEP_VISTA & 4,58 & 0,29 & 0,12 & 0,56 & $-0,103$ & 0,75 \\
\hline 1_CTASS_PAGR & 0,24 & $0,03(* *)$ & 1,50 & $0,003(* * *)$ & 0,06 & 0,63 \\
\hline 1_INTERM_FIN & 5,89 & 0,39 & 2,56 & $0,06(*)$ & 4,72 & $0,02(* *)$ \\
\hline 1_APALANC & 0,38 & $0,05(* *)$ & 1,09 & $0,02(* *)$ & 0,32 & $0,01(* * *)$ \\
\hline 1_CAPAC_COMERC & 3,81 & $0,02(* *)$ & 0,82 & 0,38 & $-0,54$ & 0,24 \\
\hline 1_LIQUIDEZ & 0,13 & 0,59 & $-0,083$ & 0,56 & 0,05 & 0,87 \\
\hline 1_HHI_DEPOS & 0,66 & 0,22 & 1,40 & $0,02(* *)$ & 2,66 & $0,01(* * *)$ \\
\hline 1_HHI_CAR_CRED & $-1,173$ & $0,02(* *)$ & $-2,024$ & $0,02(* *)$ & $-2,08$ & $0,06(*)$ \\
\hline 1_INFLAC & 0,01 & 0,91 & $-0,22$ & 0,22 & $-0,171$ & 0,22 \\
\hline 1_PIB_REĀL & $-5,12$ & $0,06(*)$ & $-7,69$ & $0,10(*)$ & $-0,727$ & 0,86 \\
\hline 1_P_PETROL & 0,33 & $0,06(*)$ & 0,07 & 0,73 & 0,02 & 0,95 \\
\hline R-cuadrado & & 0,71 & & 0,55 & & 0,60 \\
\hline R-cuadrado corregido & & 0,62 & & 0,50 & & 0,56 \\
\hline
\end{tabular}

Nota: Grado de significancia de los coeficientes diferente de $0: 10 \%(*), 5 \%(* *)$ y $1 \%(* * *)$

En relación con los bancos medianos, las cuentas por pagar presentaron una significancia del $1 \%$, el índice de intermediación financiera demostró tener una baja relación con la rentabilidad, por el contrario, el índice de apalancamiento presentó un nivel de significancia del 5\%, al igual que el nivel de concentración de depósitos y de cartera de crédito, la cual presentó una relación negativa. Al igual que con los bancos grandes, el PIB presentó un nivel de significancia del $10 \%$, pero con un coeficiente de correlación negativo.

Los bancos pequeños, las cuentas por cobrar presentaron niveles de significancia al 5\%, los depósitos a plazo tuvieron una significancia del $1 \%$, pero con una relación negativa. Los índices de intermediación financiera y apalancamiento presentaron nivel del $5 \%$ y $1 \%$ de significancia respectivamente. En cuanto a los índices de concentración, el de depósitos tuvo una alta significancia, más el de créditos tuvo una relación negativa y un nivel bajo de significancia.

En torno de la variable ROA, la Tabla 4 muestra los modelos 4,5 y 6 en los cuales se tomó la ROA de los bancos como variable dependiente, obteniendo como resultado para los bancos grandes que las cuentas por pagar presentaron niveles medios de significancia estadística, no obstante, el índice de apalancamiento presentó una relación negativa, pero una significancia del $5 \%$, al igual que el índice de concentración de créditos, pero con relación negativa. Las variables macroeconómicas PIB y precio del barril de petróleo presentaron niveles de significancia del 
10\%, más el PIB resultó tener una relación negativa.

TABLA 4

Regresiones MCO para ROA como variable dependiente

\begin{tabular}{|c|c|c|c|c|c|c|}
\hline \multirow[b]{4}{*}{ Variables independientes } & \multirow{2}{*}{\multicolumn{6}{|c|}{ Variable dependiente: I_ROA }} \\
\hline & & & & & & \\
\hline & \multicolumn{2}{|c|}{$\begin{array}{c}\text { BANCOS GRANDES } \\
\text { Modelo } 4\end{array}$} & \multicolumn{2}{|c|}{$\begin{array}{c}\text { BANCOS MEDIANOS } \\
\text { Modelo } 5 \\
\end{array}$} & \multicolumn{2}{|c|}{$\begin{array}{c}\text { BANCOS PEQUEÑOS } \\
\text { Modelo } 6\end{array}$} \\
\hline & Coef. & valor $p$ & Coef. & valor $p$ & Coef. & valor p \\
\hline 1_INVERS & 0,28 & 0,33 & $-0,12$ & 0,46 & $-0,004$ & 0,97 \\
\hline 1_CAR_CRED & $-5,866$ & 0,31 & 0,15 & 0,89 & 0,27 & 0,82 \\
\hline 1_CTAS̄_COBR & 0,09 & 0,65 & $-0,42$ & 0,15 & $-0,209$ & $0,04(* *)$ \\
\hline 1_DEP_VISTA & 4,43 & 0,29 & 0,12 & 0,57 & $-0,113$ & 0,73 \\
\hline 1_CTASS_PAGR & 0,25 & $0,03(* *)$ & 1,51 & $0,003(* * *)$ & 0,12 & 0,36 \\
\hline 1_INTERM_FIN & 5,61 & 0,40 & 2,54 & $0,06(*)$ & 4,60 & $0,03(* *)$ \\
\hline 1_APALANC & $-0,54$ & $0,02(* *)$ & 0,19 & 0,65 & $-0,374$ & $0,002(* * *)$ \\
\hline 1_CAPAC_COMERC & 3,91 & $0,02(* *)$ & 0,83 & 0,38 & $-0,423$ & 0,39 \\
\hline 1_LIQUIDEZZ & 0,15 & 0,55 & $-0,09$ & 0,54 & 0,06 & 0,86 \\
\hline 1_HHI_DEPOS & 0,65 & 0,22 & 1,40 & $0,02(* *)$ & 2,63 & $0,01(* *)$ \\
\hline 1_HHI_CAR_CRED & $-1,162$ & $0,02(* *)$ & $-2,021$ & $0,03(* *)$ & $-2,05$ & $0,08(*)$ \\
\hline 1_INFLAC & 0,01 & 0,91 & $-0,22$ & 0,23 & $-0,155$ & 0,26 \\
\hline 1_PIB_REĀL & $-5,12$ & $0,05(*)$ & $-7,69$ & $0,10(*)$ & $-0,56$ & 0,90 \\
\hline 1_P_PETROL & 0,33 & $0,06(*)$ & 0,06 & 0,74 & 0,01 & 0,97 \\
\hline R-cuadrado & & 0,79 & & 56 & & 0,42 \\
\hline R-cuadrado corregido & & 76,98 & & 1,68 & & 587,11 \\
\hline
\end{tabular}

Nota: Grado de significancia de los coeficientes diferente de $0: 10 \%(*), 5 \%(* *)$ y $1 \%(* * *)$

Los bancos medianos presentaron una significancia estadística del $1 \%$ de las cuentas por pagar con la ROA, así mismo, el índice de intermediación financiera mostró una baja influencia en la medida de rentabilidad. Por otro lado, los indicadores de concentración de mercado de depósitos y de créditos presentaron niveles bajos de significancia, no obstante, la concentración de cartera de crédito mostró una relación negativa. De las variables macroeconómicas, el PIB presentó una baja influencia respecto de la ROA.

En cuanto a los bancos pequeños, las cuentas por cobrar presentaron una relación negativa con significancia del $5 \%$ respecto de la variable dependiente ROA, el índice de intermediación financiera también tuvo una relación media y el índice de apalancamiento mostró una alta significancia. Por otro lado, la concentración de depósitos mostró una significancia del 5\%, no obstante, la concentración de créditos mostró un nivel de significancia del $10 \%$ y a su vez una relación negativa con la ROA. Las variables macroeconómicas no mostraron significancia alguna mediante el modelo de mínimos cuadrados ordinarios.

En la Tabla 5 se describen los modelos 7, 8 y 9, realizados mediante Mínimos Cuadrados Ordinarios bajo el enfoque de efectos fijos, el cual supone que las variables índice son constantes en el espacio y tiempo y el término de error incluye diferencias temporales de las observaciones (Gujarati N., 2004).

Los bancos grandes mostraron que la cuenta inversiones tuvo una baja significancia respecto de la ROE; las cuentas por pagar, así como el nivel de apalancamiento y el índice de capacidad comercial presentaron alta significancia con el $1 \%$, el índice de liquidez demostró significancia del 5\%. La concentración de los depósitos mostró una significancia del 10\%, mientras que el de créditos mostró una relación negativa con $5 \%$ de significancia. El PIB y el precio del petróleo mostraron alta significancia, nos obstante, el PIB mostró una relación negativa. 
TABLA 5

MCO con efectos fijos con la ROE como variable dependiente

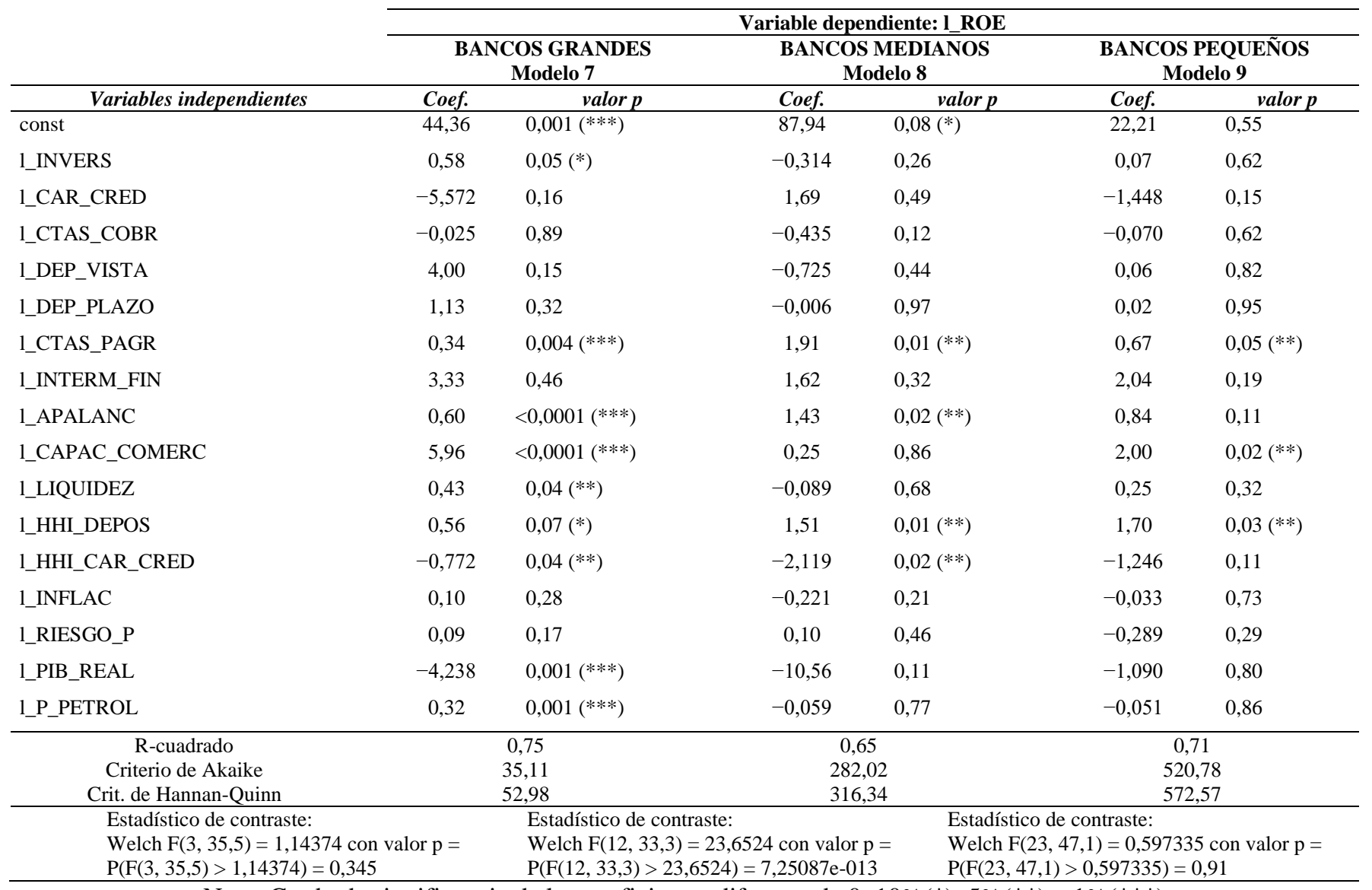

Nota: Grado de significancia de los coeficientes diferente de 0: 10\%(*), 5\%(**) y $1 \%(* * *)$

En relación con la ROE de los bancos medianos, las cuentas por pagar, el índice de apalancamiento, la concentración de los depósitos y de los créditos mostraron un nivel de significancia del 5\%. En el caso de los bancos pequeños, las cuentas por pagar, el índice de capacidad comercial y la concentración de depósitos mostraron un nivel de significancia del 5\%. Las variables macroeconómicas no presentaron relación con la rentabilidad de los bancos medianos y pequeños bajo el enfoque de efectos fijos.

La Tabla 6 muestra las regresiones bajo efectos fijos con la ROA como variable dependiente, para los bancos grandes, la cuenta inversiones tiene una significancia del $10 \%$ y las cuentas por pagar una significancia del $1 \%$. El apalancamiento y la capacidad comercial presentaron alta significancia, no obstante, el índice de apalancamiento presentó una relación negativa; el índice de liquidez mostró una significancia del 5\%. La concentración de los depósitos mostró una baja significancia, mientras que los créditos tuvieron una relación negativa con significancia del 5\%. El PIB presentó una relación negativa con la ROA y un nivel e significancia del $1 \%$, así como el precio del petróleo presentó alta significancia. 
TABLA 6

Regresiones mediante MCO con efectos fijos con la ROA como variable dependiente

\begin{tabular}{|c|c|c|c|c|c|c|}
\hline \multirow[b]{4}{*}{ Variables independientes } & \multirow{2}{*}{\multicolumn{6}{|c|}{ Variable dependiente: I_ROA }} \\
\hline & & & & & & \\
\hline & \multicolumn{2}{|c|}{$\begin{array}{c}\text { BANCOS GRANDES } \\
\text { Modelo } 10\end{array}$} & \multicolumn{2}{|c|}{$\begin{array}{c}\text { BANCOS MEDIANOS } \\
\text { Modelo } 11\end{array}$} & \multicolumn{2}{|c|}{$\begin{array}{c}\text { BANCOS PEQUEÑOS } \\
\text { Modelo } 12\end{array}$} \\
\hline & Coef. & valor $p$ & Coef. & valor $p$ & Coef. & valor $p$ \\
\hline const & 44,17 & $0,001(* * *)$ & 87,72 & $0,08(*)$ & 22,70 & 0,55 \\
\hline 1_INVERS & 0,58 & $0,05\left(^{*}\right)$ & $-0,31$ & 0,26 & 0,08 & 0,59 \\
\hline 1_CAR_CRED & $-5,41$ & 0,17 & 1,69 & 0,49 & $-1,51$ & 0,14 \\
\hline 1_CTAS_COBR & $-0,02$ & 0,91 & $-0,44$ & 0,12 & $-0,06$ & 0,66 \\
\hline 1_DEP_VISTA & 3,88 & 0,16 & $-0,73$ & 0,44 & 0,07 & 0,79 \\
\hline 1_DEP_PLAZO & 1,10 & 0,33 & $-0,006$ & 0,97 & 0,02 & 0,94 \\
\hline 1_CTAS_PAGR & 0,34 & $0,01(* * *)$ & 1,91 & $0,01(*)$ & 0,71 & $0,03(* *)$ \\
\hline 1_INTERM_FIN & 3,16 & 0,47 & 1,61 & 0,33 & 1,91 & 0,23 \\
\hline 1_APALANC & $-0,33$ & $<0,0001(* * *)$ & 0,54 & 0,32 & 0,08 & 0,87 \\
\hline 1_CAPAC_COMERC & 5,95 & $<0,0001(* * *)$ & 0,26 & 0,86 & 2,26 & $0,01(* * *)$ \\
\hline 1_LIQUIDEZ & 0,43 & $0,04(* *)$ & $-0,09$ & 0,67 & 0,26 & 0,29 \\
\hline 1_HHI_DEPOS & 0,57 & $0,07(*)$ & 1,51 & $0,01(* *)$ & 1,70 & $0,03(* *)$ \\
\hline 1_HHI_CAR_CRED & $-0,78$ & $0,04(* *)$ & $-2,12$ & $0,02(* *)$ & $-1,28$ & 0,10 \\
\hline 1_INFLAC & 0,10 & 0,30 & $-0,22$ & 0,21 & $-0,033$ & 0,73 \\
\hline 1_RIESGO_P & 0,09 & 0,17 & 0,10 & 0,47 & $-0,310$ & 0,26 \\
\hline 1_PIB_REAL & $-4,27$ & $0,00(* * *)$ & $-10,57$ & 0,11 & $-1,15$ & 0,79 \\
\hline \multirow{2}{*}{$\begin{array}{r}\text { 1_P_PETROL } \\
\text { R-cuadrado }\end{array}$} & 0,32 & $0,00(* * *)$ & $-0,06$ & 0,76 & $-0,067$ & 0,81 \\
\hline & & 0,81 & & & &, 59 \\
\hline \multicolumn{2}{|c|}{$\begin{array}{l}\text { Estadístico de contraste: } \\
\text { Welch } \mathrm{F}(3,35,6)=1,07524 \text { con valor } \mathrm{p}= \\
\mathrm{P}(\mathrm{F}(3,35,6)>1,07524)=0,372\end{array}$} & \multicolumn{2}{|c|}{$\begin{array}{l}\text { Estadístico de contraste: } \\
\text { Welch } \mathrm{F}(12,33,4)=24,364 \\
\text { con valor } \mathrm{p}=\mathrm{P}(\mathrm{F}(12,33,4)>24,364)=\end{array}$} & \multicolumn{3}{|c|}{$\begin{array}{l}\text { Estadístico de contraste: } \\
\text { Welch } \mathrm{F}(23,47,0)=0,616449 \text { con valor } \mathrm{p}= \\
\mathrm{P}(\mathrm{F}(23,47,0)>0,616449)=0,895\end{array}$} \\
\hline
\end{tabular}

Nota: Grado de significancia de los coeficientes diferente de $0: 10 \%(*), 5 \%(* *)$ y $1 \%(* * *)$

En relación con la ROA de los bancos medianos, las cuentas por pagar presentaron una significancia del 10\%, mientras que la concentración de los depósitos y los créditos una significancia del $5 \%$, teniendo los créditos una relación negativa. En el caso de los bancos pequeños, las cuentas por pagar señalan una significancia del $5 \%$, al igual que la concentración de depósitos; el índice de capacidad comercial mostró una significancia del $1 \%$.

Se realizaron estimaciones de Mínimos Cuadrados Ordinarios bajo el enfoque de efectos aleatorios, las cuales se muestran en la Tabla 7 se muestran las regresiones de $\mathrm{MCO}$ para la variable dependiente ROE bajo efectos aleatorios indica una significancia estadística del $10 \%$ para la cuenta inversiones, para el apalancamiento y la capacidad comercial presentó una alta significancia con el 1\%. La concentración de los créditos mostró una alta significancia estadística con una relación negativa. El PIB y el precio del petróleo tuvieron significancia del $1 \%$, pero PIB mostró una relación negativa. 
TABLA 7

MCO con efectos aleatorios con la ROE de los bancos como variable dependiente

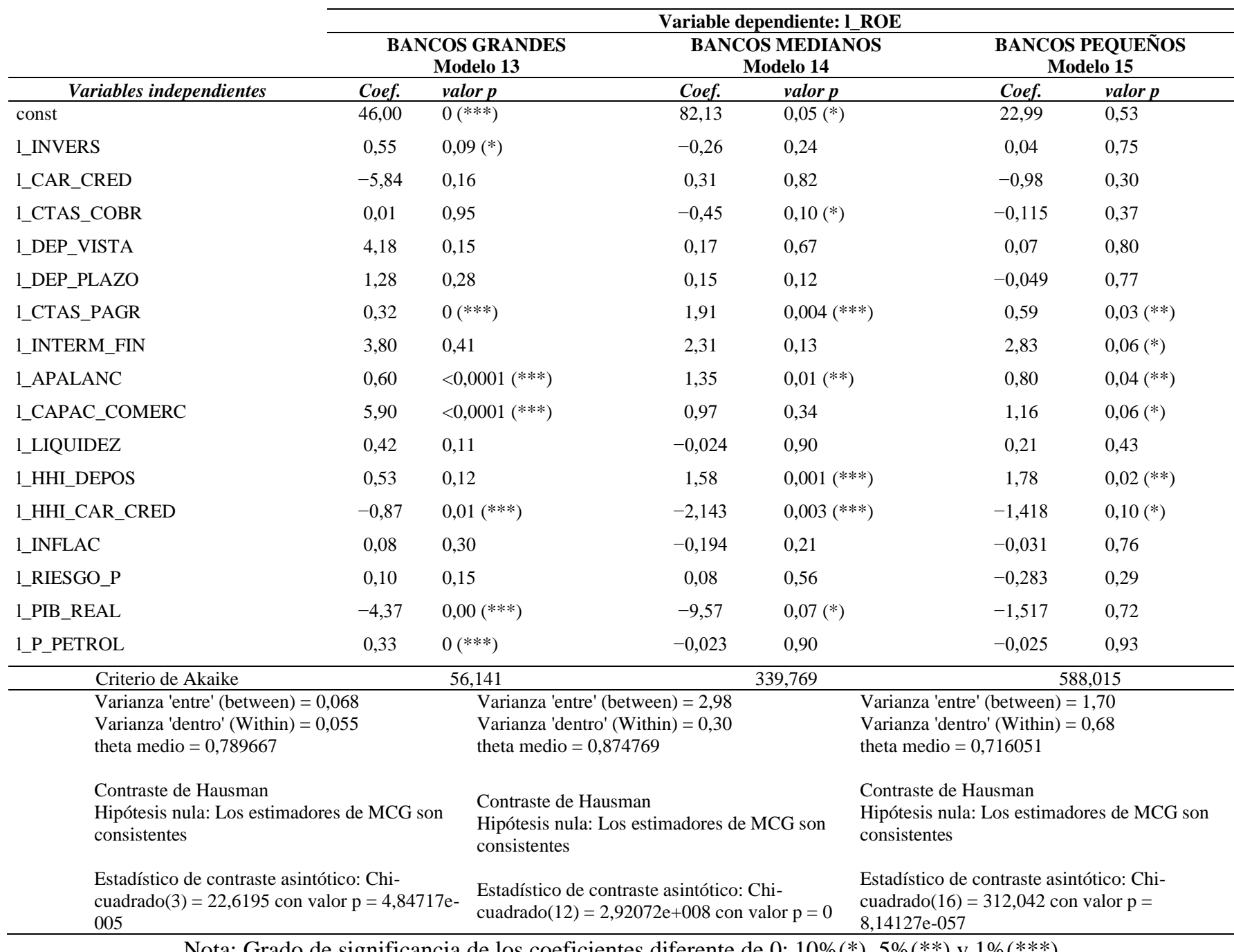

Por su parte, los bancos medianos mostraron una baja significancia y una relación negativa de las cuentas por cobrar con la ROE, sin embargo, las cuentas por pagar tuvieron una significancia del $1 \%$ y el apalancamiento del $5 \%$. La concentración de depósitos y de créditos mostró alta significancia con un grado de $1 \%$ en ambos casos, mientras que el PIB presentó una baja significancia con un nivel de $10 \%$ y una relación negativa respecto de la ROE.

En el caso de los bancos pequeños, el índice de intermediación financiera y el de capacidad comercial mostraron un grado de significancia bajo con $10 \%$, las cuentas por pagar y el grado de apalancamiento presentaron una significancia del 5\%. El índice e concentración para los depósitos tuvo una significancia del 5\%, sin embargo el de créditos tuvo una significancia baja con el $10 \%$ pero con relación negativa con la ROE.

La Tabla 8 muestra las regresiones de $\mathrm{MCO}$ tomando como variable dependiente la ROA de los bancos y bajo el enfoque de efectos aleatorios, obteniendo como resultado para los bancos grandes un nivel medio de significancia de la cuenta inversiones, de la concentración de la cartera de créditos, el PIB y el precio de barril de petróleo, no obstante, los créditos y el PIB mostraron una relación negativa. Las cuentas por pagar mostraron una significancia el 10\%, al igual que el apalancamiento con una relación negativa. El índice de capacidad comercial presentó una alta significancia estadística. 
TABLA 8

MCO con efectos aleatorios con la ROA de los bancos como variable dependiente

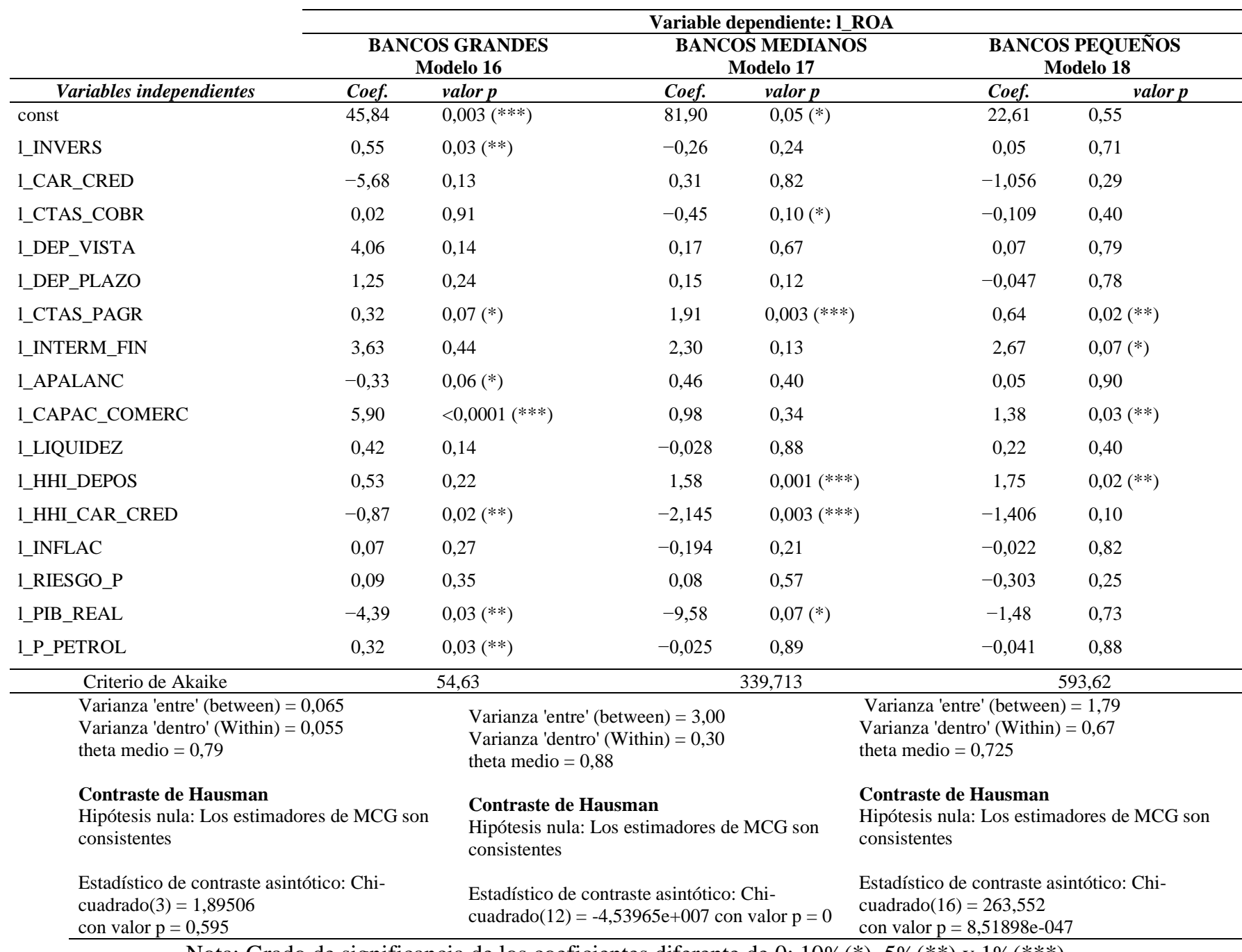

Nota: Grado de significancia de los coeficientes diferente de $0: 10 \%(*), 5 \%(* *)$ y $1 \%(* * *)$

Los bancos medianos mostraron que las cuentas por cobrar y el PIB tienen relación negativa con baja significancia estadística respecto de la ROA. Así mismo, las cuentas por pagar, la concentración de depósitos y la de crédito mostraron alta significancia con el $1 \%$, sin embargo, esta última variable mostró una relación negativa. En el caso de los bancos pequeños, las cuentas por pagar, la capacidad comercial y la concentración de los depósitos presentaron una significancia estadística del $5 \%$ en relación con la ROA. El índice de intermediación financiera mostró una baja significancia.

En la Tabla 7 y la Tabla 8 se incluyó como parte de los resultados de las regresiones bajo efectos aleatorios el test de Hausman (1978), que según Gujarati (2004) compara las estimaciones realizadas mediante los modelos bajo efectos fijos con los de efectos aleatorios y de acuerdo a su valor-p determinar el enfoque que explica mejor la relación de las variables con los datos empleando una estructura de datos de panel.

En este sentido, tomando como variable dependiente la ROE para los bancos grandes, el enfoque idóneo es el de efectos fijos, puesto que su valor p es más cercano a cero, en comparación con el de efectos aleatorios. Para los bancos medianos, conviene el enfoque de efectos aleatorios, puesto que su p-value es igual a cero. 
Para evaluar los bancos pequeños también es idónea la utilización del enfoque de efectos fijos.

Para las regresiones tomando como variable dependiente la ROA, el modelo de efectos fijos es el más idóneo para explicar la relación de las variables para los bancos grandes. Para los bancos medianos, el enfoque de efectos aleatorios es el más idóneo, con un valor-p igual a cero. En relación con los bancos pequeños, el modelo que mejor explica la relación de las variables es el de efectos aleatorios, que también posee un valor menor cercano a cero.

\section{Discusión}

Los resultados obtenidos por Athanasoglou, Brissimis, y Delis (2008) que analizaron la ROE y ROA y señalaron que la inflación afecta el rendimiento del sector bancario, difieren con los resultados de la presente investigación, puesto que se determinó que la rentabilidad en términos de ROE y ROA se ve influenciada por el PIB y por el precio del barril de petróleo, en el caso de los bancos grandes y medianos.

Además, los niveles de inflación no mostraron relación alguna con las medidas de rentabilidad lo cual se alinea con el estudio de García-Herrero, Gavilá, y Santabárbara (2009) que señalaron que los niveles de inflación no inciden en el desempeño de los índices de rentabilidad de los bancos, sino, los créditos vencidos.

A su vez, concuerda con los resultados de Curak, Poposki y Pepur (2012), Osuagwu (2014), Abduh y Omar (2013), y Ashraf, Haider, y Sarwar (2017), quienes concluyeron que la rentabilidad de los bancos está determinada por factores como, los niveles de solvencia, liquidez, concentración de mercado y el crecimiento del PIB, dado que en el presente estudio el PIB demostró tener relación con la ROE y ROA de los bancos medianos y grandes, así como también el índice de liquidez de los bancos grandes y las medidas de concentración de depósitos y de créditos en la banca privada en general.

También se obtuvo como resultado que el crecimiento del PIB Afecta la ROA y ROE de bancos medianos y grandes, lo cual se opone a los del estudio de Chidozie y Ayadi (2017) que evidenciaron que el crecimiento del PIB no afecta la rentabilidad bancaria en Nigeria. No obstante, convergen en que y que el precio del barril de petróleo afecta la rentabilidad del sector financiero, puesto que para la banca privada ecuatoriana el precio del barril de petróleo afecta principalmente la ROE y ROA de los bancos grandes.

En cuanto al nivel de apalancamiento, éste afecta principalmente a la ROE de los bancos grandes y medianos y la ROA de los bancos grandes, lo cual es opuesto a lo indicado por Jara Quevedo et al., (2018), puesto que los bancos pequeños no se vieron afectados por esta variable en el presente estudio. No obstante, coincide en que el PIB afecta la pequeña banca privada, así como también el índice de intermediación financiera.

\section{CONCLUSIONES}

En el presente estudio los factores internos como las cuentas por pagar resultaron estar relacionados con la rentabilidad de la banca privada en general, lo que implica que las obligaciones de los bancos tienen un papel importante en la rentabilidad, sin importar el tamaño del banco. Así también la cuenta inversiones y las cuentas por cobrar tiene relevancia respecto de la ROE de los bancos.

Además, se identifica que la capacidad comercial, influye en la ROE de los bancos grandes y pequeños, $\mathrm{Y}$ los niveles de liquidez afectan mayormente la ROE de los bancos grandes y las cuentas por cobrar influyen en la ROE de los bancos medianos. Los niveles de apalancamiento se relacionan con la ROA de los bancos, lo que implica un efecto del endeudamiento con fondos externos en la rentabilidad sobre el patrimonio de los bancos privados medianos y grandes.

En cuanto a los niveles de concentración medidos con el índice de Herfindahl Hirschman, la concentración de depósitos se relaciona con la ROE de los bancos privados sin importar su tamaño, no obstante, la concentración de los créditos está relacionada con la ROE de bancos 
grandes y medianos, mas no con la rentabilidad sobre patrimonio de bancos pequeños. En el caso de la ROA, los niveles de concentración de créditos y depósitos se relacionan con la rentabilidad de activos de bancos grandes y medianos. La ROA de bancos pequeños solo está relacionada con la concentración de los depósitos.

Entre las variables macroeconómicas como el PIB mayormente se relacionan con la ROE de los bancos grandes y medianos. Por otro lado, las variaciones en el precio del barril de petróleo afectan la economía nacional en general. Sin embargo, se demostró en el presente estudio que esta variable macroeconómica tiene alta influencia en la rentabilidad de los bancos.

\section{REFERENCIAS}

Alvarado V., V., 2016. Ingeniería de costos. Azcapotzalco: Grupo Editorial Patria.

Asamblea Nacional Constituyente, 2008. Art.- 283.. Montecristi(Manabí): s.n.

Ashraf, M., Haider, Z. \& Sarwar, M. B., 2017. Bank Specific and Macroeconomic Determinants Impact on Banks Profitability: Evidence from Asian Countries. International Journal of Sciences: Basic and Applied Research (IJSBAR), 33(3), pp. 187-199.

ASOBANCA, 2017. Evolución de la banca privada Ecuatoriana, s.l.: s.n.

Athanasoglou, P. P., Brissimis, S. N. \& Delis, M. D., 2008. Bank-specific, industry-specific and macroeconomic determinants of bank profitability. Journal of International Financial Markets, Institutions and Money, Abril.pp. 121-136.

Baumol, W. \& Blinder, A., 2009. Economics: Principles and Policy. Mason(Ohio): Cengage Learning .

BCE, 2021. Banco Central del Ecuador. [En línea] Available at:

https://sintesis.bce.fin.ec/BOE/OpenDocument/20110 10922/OpenDocument/opendoc/openDocument.faces ? $\log$ onSuccessful=true \&share $I d=2$

Camino-Mogro, S. \& Armijos-Bravo, G., 2018. Assessing competition in the private banking sector in Ecuador: an econometric approach with the
Panzar-Rosse model. Cuadernos de economía, 29 Octubre, 41(117), pp. 225-307.

Chidozie, U. E. \& Ayadi, F. S., 2017. Macroeconomy and Banks' Profitability in Nigeria. African Research Review, Abril, 11(46), pp. 121-137.

Chiriboga R., L. A., 2010. Sistema Financiero Nacional. En: Sistema Financiero. Quito, Ecuador: PUBLIGRÁFICAS JOKAMA, pp. 6 - 10.

Congreso Nacional del Ecuador, 2000. Ley para la transformación económica del Ecuador.

Quito(Pichincha): s.n.

Cruz Rodríguez, C., 2015. RESOLUCiÓN No. SB2015-66S. s.1.:s.n.

Curak, M., Poposki, K. \& Pepur, S., 2012.

Profitability Determinants of the Macedonian Banking Sector in Changing Environment. Procedia Social and Behavioral Sciences, Junio, Volumen 44, pp. 406-416.

Dietrich, A. \& Wanzenried, G., 2010. Determinants of bank profitability before and during the crisis: Evidence from Switzerland. Journal of International Financial Markets, Institutions \& Money, 7 Diciembre, 21(3), pp. 307-327.

García O., M., Martínez G., F. \& Fernández G., E., 2018. Mercado de renta variable: Análisis de títulos. Madrid: Paraninfo S.A..

García-Herrero, A., Gavilá, S. \& Santabárbara, D., 2009. What explains the low profitability of Chinese banks?. Journal of Banking \& Finance, pp. 20802092.

Gibson, C. H., 2012. Financial Reporting and Analysis. Mason(Ohio): Cengage Learning.

Gómez G., J., Uribe, J. \& Piñeros, H., 2009.

Determinantes de la Rentabilidad de los Bancos en Colombia: ¿Importa la Tasa de Cambio?. Borradores de Economía, Issue 556.

Gujarati N., D., 2004. Basic Econometrics. s.l.:The McGraw Hill.

Hausman, J. A., 1978. Specification Tests in Econometrics. Econometrica, Noviembre, 46(6), pp. 1251-1271.

Jara Quevedo, G., Sánchez Giler, S., Bucaram Leverone, R. \& García Regalado, J., 2018. 
ANÁLISIS DE INDICADORES DE

RENTABILIDAD DE LA PEQUEÑA BANCA PRIVADA EN EL ECUADOR A PARTIR DE LA DOLARIZACIÓN. Compendium - Cuadernos de Economía y Administración, 24 diciembre, 5(12), pp. 54-76.

Jones, C. I., 2009 . Macroeconomía. Barcelona: Antoni Bosch S.A. .

Muhammad, A. \& Omar, M. A., 2013. Profitability Determinants of Islamic and Conventional Banks in Malaysia: A Panel Regression Approach.

Terengganu International Finance and Economics Journal, Enero, 3(1), pp. 1-7.

Noboa S., M., 2008. Banca y Moneda. s.1.:CODEU.

Osuagwu, E. S., 2014. Determinants of Bank Profitability in Nigeria. International Journal of Economics and Finance, 25 Noviembre , 6(12), pp. 46-61.

Pampillón, F., De la Cuesta, M. \& Ruza, C., 2012. Principales elementos del sistema financiero. En: Introducción al sistema financiero. Madrid, España: UNED Publicaciones, pp. 191-200.

Parkin, M., Esquivel, G. \& Muñoz, M., 2007. Macroeconomía: versión para latinoamérica. México D.F. : Pearson Education.

Registro Oficial No. 475, 1994. REGLAMENTO A LA LEY GENERAL DE INSTITUCIONES DEL SISTEMA FINANCIERO. Quito(Pichincha): s.n.

Romero A., P., 2004-2005. CRISIS BANCARIA EN ECUADOR: CAUSAS Y POSIBLES

SOLUCIONES. Instituto Ecuatoriano de Economía Política.

Romero-Galarza, A., Flores-Sánchez, G., ReyesZambrano, B. \& Campoverde-Campoverde, J., 2020. Evaluación de la concentración y competencia delsector bancario ecuatorianoen el periodo 20062018. Boletín de Coyuntura, 5 Marzo, Issue 24, pp. 4-12.
Solano, J., Camino-Mogro, S. \& Armijos-Bravo, G., 2020. Intra-banking competition in Ecuador: new evidence using panel data approach. Journal of Economics, Finance and Administrative Science, 25(50), pp. 295-319.

Superintendencia de Bancos, 2017. Normativa Catálogo único de cuentas. [En línea]

Available at:

https://www.superbancos.gob.ec/bancos/catalogo-decuentas/

Superintendencia de Bancos, 2021. Catálogo de Cuentas - Sistema Financiero. [En línea]

Available at:

https://www.superbancos.gob.ec/bancos/wpcontent/uploads/downloads/2020/06/CUC-plancuentas-pasivos 27-sept-17.pdf [Último acceso: 3 Marzo 2021].

Toscanini, M., Lapo-Maza, M. \& Bustamante, M. A., 2020. La dolarización en Ecuador: resultados macroeconómicos en las dos últimas décadas. Información Tecnológica, Octubre, 31(5), pp. 129138.

Uzcátegui-Sánchez, C., Camino-Mogro, S. \& MoranCruz, J. C., 2018. Estructura de mercado del sistema bancario ecuatoriano: concentración y poder de mercado. CUMBRES, Enero - Junio, 4(1), pp. 49-62.

Valdez M., M., Jara Q., G., Zurita V., S. \& Massuh A., O., 2020. Relación entre el crédito financiero y de proveedores con ingresos de empresas del sector agropecuario ecuatoriano. X-Pedientes Económicos, Enero-Abril, 4(8), pp. 63-77.

Vera-Gilces, P., Camino-Mogro, S., OrdeñanaRodríguez, X. \& Cornejo-Marcos, G., 2020. A look inside banking profitability: Evidence from a dollarized emerging country. The Quarterly Review of Economics and Finance, Febrero, Volumen 75, pp. 147-166. 
APÉNDICE A

Descripción de las variables

\begin{tabular}{|c|c|c|c|}
\hline Variables & Descripción & Etiqueta & $\begin{array}{r}\text { Relación } \\
\text { esperada }\end{array}$ \\
\hline \multicolumn{4}{|c|}{ Variables Dependientes } \\
\hline Rentabilidad Patrimonial & Resultados/Patrimonio & ROE & \\
\hline Rentabilidad de Activos & Resultados/Activos & ROA & \\
\hline \multicolumn{4}{|c|}{ Variables independientes } \\
\hline \multicolumn{4}{|l|}{ Cuentas de los bancos } \\
\hline Inversiones & Inversiones realizadas por los bancos & INVERS & $+/-$ \\
\hline Cartera de Créditos & Total de la cartera de crédito & CAR_CRED & $+/-$ \\
\hline Cuentas por Cobrar & Cuentas por cobrar de los bancos & CTAS_COBR & $+/-$ \\
\hline Depósitos a la vista & Total Depósitos a la vista & DEP_VISTA & $+/-$ \\
\hline Depósitos a plazo & Total depósitos a plazo & DEP_PLAZO & $+/-$ \\
\hline Cuentas por pagar & Deudas de los bancos & CTAS_PAGR & $+/-$ \\
\hline \multicolumn{4}{|l|}{ Indicadores financieros } \\
\hline Intermediación Financiera & Cartera bruta/Depósitos totales & INTERM_FIN & $+/-$ \\
\hline Apalancamiento & Total Pasivos/Patrimonio & APALANC & $+/-$ \\
\hline Capacidad Comercial & Cartera total/Activo total & CAPAC_COMERC & $+/-$ \\
\hline Liquidez inmediata & Fondos disponibles/Depósitos a corto plazo & LIQUIDEZ & $+/-$ \\
\hline \multicolumn{4}{|c|}{ Indicador de Concentración de mercado - Herfindahl Hirschman (HHI) } \\
\hline Concentración de depósitos totales & HHI aplicado a los depósitos totales & HHI_DEPOS & $+/-$ \\
\hline $\begin{array}{c}\text { Concentración de Cartera de } \\
\text { Créditos } \\
\end{array}$ & HHI aplicado al total de cartera de créditos & HHI_CAR_CRED & $+/-$ \\
\hline \multicolumn{4}{|l|}{ Indicadores macroeconómicos } \\
\hline Inflación & Inflación anual & INFLAC & $+/-$ \\
\hline Riesgo País & Nivel anual del Riesgo país & RIESGO_P & $+/-$ \\
\hline Producto Interno Bruto Anual & Total del PIB real anual & PIB_REAL & $+/-$ \\
\hline Precio del Petróleo & Precio del barril de petróleo & P_PETROL & $+/-$ \\
\hline
\end{tabular}

